\title{
PERCEPCIONES DE LOS AGENTES COMUNITARIOS SOBRE LA PARTICIPACIÓN COMUNITARIA EN UNA COMUNIDAD DEL CALLAO-PERÚ, 2020
}

\section{PERCEPTIONS OF COMMUNITY AGENTS ON COMMUNITY PARTICIPATION IN A COMMUNITY OF CALLAO-PERU, 2020}

\author{
Ronald Espíritu Ayala Mendívil ${ }^{1}$, Sonia Shishido Sánchez ${ }^{1}$ \\ ${ }^{1}$ Universidad Nacional Mayor de San Marcos. Facultad de Medicina. Lima. Perú.
}

\section{INFORMACIÓN DEL ARTÍCULO}

Historia del artículo Recibido: 28/11/2021

Aprobado: $15 / 12 / 2021$

Publicado: 31/12/2021

Autor corresponsal Ronald Espíritu Ayala Mendívil ream37339@yahoo.com

Financiamiento Autofinanciado

Conflictos de interés Los autores declaran no tener conflictos de interés

\section{Citar como}

Ayala Mendívil RE, Shishido

Sánchez S. Percepciones de los agentes comunitarios sobre la participación comunitaria en una comunidad del Callao-Perú, 2020. Ágora Rev. Cient. 2021; 08(02): 8894. DOI: https://doi.org/10.21679/ arc.v8i2.224

\begin{abstract}
RESUMEN
Objetivo: Explorar las percepciones de los agentes comunitarios sobre la participación comunitaria en la comunidad de Bellavista-Callao, en el mes de febrero de 2020. Materiales y métodos: El presente estudio es de enfoque cualitativo de diseño fenomenológico y exploratorio. La población del estudio estuvo conformada por 8 agentes comunitarios activos de la comunidad de Bellavista-Callao. Se entrevistaron a los agentes, previo consentimiento informado. Se procesó la información mediante el programa Atlas.ti v.9, se transcribieron las grabaciones y se identificaron categorías, con subcategorías y códigos. Resultados: Se identificaron 3 categorías principales: Percepciones sobre la definición de Participación Comunitaria en Salud, Percepciones sobre la Operativización de la participación y Percepciones sobre la Ejecución de la participación, con subcategorías donde las entrevistadas refirieron diversas percepciones sobre participación comunitaria, compromiso con la participación comunitaria; operativización de la participación, visitas domiciliarias; ejecución de la participación y relación de lo asistencial con la participación comunitaria. Se interpretaron las relaciones existentes entre las diversas categorías, subcategorías y códigos identificados. Conclusiones: El tipo de participación de la comunidad evidenciada es pasiva o pseudo-participación. Se remarca la injerencia político partidaria en temas de salud. Se reconoce la intervención de los agentes comunitarios, por su labor con motivación, vocación de servicio y empatía, a su vez, se recomienda fortalecer en el trabajo las capacidades que permitan reorientar el trabajo comunitario además del esfuerzo que realiza los agentes haca la comunidad, modificando la concepción como asistencial referido a los agentes comunitarios involucrados.
\end{abstract}

Palabra claves: Participación de la comunidad; Agentes comunitarios; Percepción (Fuente: DeCS).

\begin{abstract}
Objective: To explore the perceptions of community agents about community participation in the Bellavista-Callao community, in February 2020. Materials and methods: This study has a qualitative approach of phenomenological and exploratory design. The study population consisted of 8 active community agents from the Bellavista-Callao community. Agents were interviewed with prior informed consent. The information was processed using the Atlas.ti v.9 program, the recordings were transcribed and categories were identified, with subcategories and codes. Results: 3 main categories were identified: Perceptions on the definition of Community Participation in Health, Perceptions on the Operationalization of participation and Perceptions on the Execution of participation, with subcategories where the interviewees referred various perceptions on community participation, commitment to participation community; operationalization of participation, home visits; execution of the participation and relation of the assistance with the community participation. The relationships between the various categories, subcategories and identified codes were interpreted. Conclusions: The type of community participation evidenced is passive or pseudo-participation. Political party interference in health issues is highlighted. The intervention of community agents is recognized, for their work with motivation, vocation of service and empathy, in turn, it is recommended to strengthen at work the capacities to carry out reorient community work in addition to the effort made by agents towards the community, modifying the conception as assistance referred to the community agents involved.
\end{abstract}

Keywords: Community involvement; Community workers; Perception (Source: DeCS). 
"Participación". Se puede definirlo como Intervención en un acto, una actividad que persigue una meta u objetivo (1). También puede definirse como el proceso en el que una comunidad interviene en la solución de sus propios problemas que acorde a sus experiencias pueden llegar hasta la toma de decisiones que permitan llegar a resultados medibles y deseables para la misma comunidad ${ }^{(2,3)}$. En ese sentido, la población asume sus responsabilidades en el marco de su salud individual, familiar y colectiva, identificando necesidades problemas y planteando soluciones ${ }^{(4,5)}$. Además, Cassetti V. y colaboradores (6), lo definen como el proceso mediante el cual diferentes agentes comunitarios se relacionan de manera directa o indirecta en alguna de las fases del proceso, mediante el uso de diversos métodos tanto de consulta, empoderamiento o colaboración. Ante ello emerge la figura de los Agentes comunitarios, como representantes de la comunidad que poseen ciertas características y cualidades descritas por los autores y que intervengan en alguna parte o en todo el proceso de planificación local ${ }^{(6-8)}$, que permiten que tengan cierto nivel de arraigo en la comunidad a la que representan y puedan ejercer una labor de soporte y coordinación intersectorial muy estrecha, vinculándose con los establecimientos de salud ${ }^{(9)}$.

De tal manera, que los agentes comunitarios al ser los responsables para la relación con los establecimientos de la salud (10), ya que va a permitir mejorar los accesos de atención en la comunidad, donde fomenten una mejor atención en salud y a la vez comunitario ${ }^{(11,12)}$.

En la comunidad de Bellavista-Callao, la labor comunitaria se muestra muy limitada, motivo por el cual se ha realizado la presente investigación. Se formuló el siguiente problema: ¿Cuáles son las percepciones de los agentes comunitarios sobre la participación comunitaria en la jurisdicción del distrito de Bellavista-Callao, en el mes de febrero del 2020 ?

Su objetivo fue explorar las percepciones de los agentes comunitarios sobre la participación comunitaria en la jurisdicción del distrito de Bellavista-Callao, en el mes de febrero del 2020. Esta investigación se justifica por cuanto la participación comunitaria en salud está plenamente reconocida como un elemento fundamental para el logro de los objetivos comunales, y esta debe relacionarse con otros factores que permitan el adecuado empoderamiento de la población.

\section{MATERIALES Y MÉTODOS}

\section{Enfoque y diseño de estudio}

Investigación de tipo cualitativa de diseño fenomenológico y exploratorio por cuanto reúne las características de abordaje desde la lógica interna del sujeto investigado, comprende la realidad que es vista como subjetiva, compleja, heterogénea ${ }^{(13)}$. Brinda su énfasis en lo individual y particular ${ }^{(14)}$.

\section{Población de estudio}

Se aplicó una entrevista a profundidad a 08 agentes comunitarios de lascuales 3 de ellastrabajan articuladamente con el Centro de Salud "Perú Korea Bellavista", y las otras 5 personas son miembros de organizaciones sociales de base tales como comedores populares y vasos de leche de la jurisdicción, que cumplen con los siguientes criterios de inclusión: miembro activo de una organización social de base, persona que resida en la comunidad de Bellavista, tiempo de residencia mayor o igual a 2 años, persona que acepte participar en el estudio. Asimismo, se cumplieron los siguientes criterios de exclusión siguientes: Persona que pertenezca a alguna organización social de base de otras jurisdicciones, tiempo de residencia menor a 2 años, persona que no acepte participar en el estudio. Se identificaron 3 categorías a saber: definición de participación comunitaria, operativización de la participación, ejecución de la participación. Cada entrevista tuvo una duración individual aproximada entre 45 minutos a 01,5 horas y se aplicó hasta alcanzar el punto de saturación teórica, realizándose en un ambiente agradable, de preferencia en su mismo lugar de labores o en su domicilio, mediante grabación de audio. Para el análisis de los datos, se revisaron los apuntes y grabaciones, se utilizó el programa Atlas.ti v.09.

\section{Consideraciones éticas}

Los autores declaran haber leído y respetado la Declaración de Helsinki, no se vulneraron los aspectos éticos de ninguna persona incluida en el estudio, manteniendo la respectiva confidencialidad y respeto, se procedió a ejecutarla previo consentimiento informado de la persona, garantizando la confidencialidad de las grabaciones realizadas. Se respetaron los principios bioéticos en la investigación ${ }^{(15,16)}$.

\section{RESULTADOS}

Se obtuvieron las siguientes percepciones acorde a cada categoría identificada:

\section{1) Percepciones sobre la definición de participación comunitaria en salud:}

En relación a la definición de participación comunitaria en salud, las entrevistadas refieren que ello implica disponer de su tiempo para realizar un trabajo con el fin de servir al resto, pero que ello no resulta si la misma comunidad no tiene la predisposición de aceptar el trabajo que los agentes 
comunitarios realizan, y que valoren las coordinaciones que realizan con el establecimiento de salud. Se resalta la capacidad organizativa con las autoridades locales para el fomento de la participación:

"A ver, una participación, digamos así, comunitaria es el trabajar, para mí, sin tiempo, bueno para disponer de mi tiempo libre, no tengo horario, yo no tengo horario, a veces yo misma digo ni yo dispongo de mi tiempo, porque si me llaman para servir o porque me necesitan en algún lado, lo hago, salgo en el instante, o sea dejó de hacer lo que estoy haciendo" (E1, L34).

"La participación comunitaria sería que la comunidad participe en estos temas, que participe de repente recibiendo los consejos, las sugerencias, los avisos todo concerniente a los temas de salud, a las difusiones o de repente si le toca un control pues hay que asistir, si le toca llevar al niño no dejar de asistir, que no pierdan su fecha de control" (E4, L29).

"Ahora está mucho mejor, más organizado, porque antes no había esas charlas en cambio ahora recién tenemos las charlas, nos Ilaman a capacitación (...) Tanto las juntas vecinales con el vaso de leche toditas nos informamos, nos organizamos para participar" (E7, L141).

Por otro lado, siguen las opiniones relacionadas a la participación en campañas de salud como parte del concepto de la Participación comunitaria, estando supeditada su participación a la cercanía domiciliaria del lugar de la campaña y revelando otra limitante en la despreocupación de parte de los pobladores por su propio estado de salud actual. A pesar de ello, se releva el dinamismo que de por sí le dan a la participación como eje clave para motivar al resto:

"Bueno la gente más va de alrededor porque están cerca ¿no? porque de otra zona no vienen, aparte de que dependiendo eso sí puede ser libre, por decirlo, que vengan de otra zona" (E1, L121).

"En cuanto a la salud, pienso que las campañas, ¿por qué? Porque si no se enteran, si no sabe Ud. no va a salir de casa para ir a, que yo le diga vaya al centro, ¿para qué? Si no tienen una consulta, no se preocupan en sí por ahondar un poquito más en su salud (...)" (E1, L127).

"Opino que, si debe de haber participación, pero como le digo con temas que sean interesantes a la comunidad hay mucha gente mayor que pueden ir a escuchar, pero no vamos a ir a escuchar (...) Dar temas que a la gente le ponga pilas (...), pero tiene que ser talleres pilas para que la gente participe, para que la gente esté dinámica y no para que la gente se duerma" (E8, L27).

Se resalta la injerencia política que en la comunidad está presente de una manera muy enraizada en la mentalidad de la población:

"Concientizar un poquito más al resto de personas ¿no? para que en adelante participen, porque parece que lo han mentalizado de que todo es en base a política" (E4, L44).

En relación al Compromiso con la participación comunitaria en salud, las entrevistadas denotan actitudes favorables que demuestran que pueden organizar sus propias labores diarias en sus familias, y que pueden organizar su tiempo en pro de garantizar su participación activa, refiriendo que le encuentran un "gusto agradable" por la labor que realizan, sabiendo escuchar a la gente y como refieren, "tomando en serio su labor" y "voluntad de trabajo":

“(...) No me hago problemas, entonces y es el trabajar, como quien dice hacer el bien sin mirar a quien ¿no? $\mathrm{Y}$ a donde vaya porque no tengo miedo de llegar al rincón más bajo ni tampoco tengo el miedo de rozar con la gente ¿no? (...) Nosotras, las demás hemos sido convocadas igual, hemos sido convocadas para participar, nos dieron una capacitación o una charla para mostrarnos el trabajo que vamos hacer, para explicarnos y salir a campo (...9 Todas han tenido y sé que tienen voluntad, tienen espíritu del trabajo eso si les gusta, nos gusta lo que hacemos, en realidad nos gusta lo que hacemos (...)" (E1, L34-40).

"Digamos, le digo, no sé, es tomármelo de repente como forma personal, tomar muy en serio en sí la labor que estoy realizando, ya no me veo yo, ni me ven mucha gente al llegar a casa por ejemplo ¿no? (...) Porque me gusta escuchar a la gente, me entiende, a veces yo misma me comprometo (...) entonces es tomar en serio en sí nuestra labor" (E1, L55).

\section{2) Percepciones sobre la operativización de la participación:}

En relación a la operativización de la participación, se han recogido diversas expresiones sobre el concepto de empoderamiento que poseen las entrevistadas, entre las que destacan deben estar fortalecidas con capacitaciones, que les permitan ejercer una labor contundente en comunidad, garantizando el seguimiento de casos y asegurándose que los pacientes sigan las indicaciones brindadas por el establecimiento de salud, todo esto tiene un fuerte componente asistencialista de la atención:

"Por empoderamiento yo entiendo de que las personas deben saber que tienen que ir a un control hacer seguimiento, continuar el tratamiento, no abandonarlo como la TBC comienzan y ya cuando están por cuatro o cinco meses y de ahí lo dejan (...)" (E3, L55).

"El empoderamiento es de repente estar fortalecido a través de las capacitaciones, de charlas, de consejos, de capacitaciones que nos dan (...)" (E4, L47).

"El empoderamiento nosotros lo entendemos cuando tenemos conocimiento herramientas para poder estar empoderadas y saber hacia dónde vamos (...)" (E8, L42).

En relación a la definición que poseen de Visita domiciliaria, las entrevistadas refieren, que dicha actividad garantiza el seguimiento de pacientes que realiza el 
establecimiento de salud, teniendo la oportunidad de realizar un abordaje de otras problemáticas, priorizando nuevamente el aspecto asistencialista de la atención:

"Bueno para nosotros la visita en sí es que vamos para hablar con, digamos, con quién nos corresponde aquí en cuanto a la visita esa para los niños, tenemos que hacer por ejemplo las consulta sobre el programa o sobre el tratamiento de la criatura e informarnos si nos quieren manifestar alguna otra preocupación o algún otro interés que tengan ya en casa, alguna otra problemática" (E1, L67).

"También he logrado hacer visitas domiciliarias a pacientes que no pueden llegar al centro, salimos en movilidad entonces hacemos el control en el domicilio, pero también lo hacíamos en el mismo centro a las personas que van y hacen su control o para empezar su tratamiento en hipertensión" (E1, L13).

\section{3) Percepciones sobre la ejecución de la participación:}

En relación a la ejecución de la participación, las entrevistadas refieren que ello se lleva a cabo gracias a un trabajo de coordinación intersectorial en especial con las escuelas que debe estar fortalecido a fin de contribuir a resolver los problemas de salud de la comunidad, con prioridad a la población infantil, relacionándolo con la articulación con las autoridades locales como de la Municipalidad I distrital o provincial:

"Yo creo que el Perú Korea tiene buenas intenciones, pero tratan, pero no queda, por lo que a los colegios deberían ir ellos (...) deberían darles información a los colegios, enseñarles a los niños ya que la mayor parte están solos porque los padres trabajan (...) El tema es la coordinación de horarios, programarlo un domingo, pero como le digo que la gente está tan apurada vive tan aprisa, viene de su casa a descansar prácticamente y se olvida de todo." (E5, L102-109).

"Primeramente, es el vaso de leche de la municipalidad, ellos nos informan cualquier reunión que tengan, de cualquier charla, ellos son los que más o menos los que convocan en la municipalidad de Bellavista (...). También nos ayudan en orientarnos en que vayamos al seguro, o a este otro, también nos aconseja la presidente sobre eso porque nos ayuda en hablar con los doctores y nos pasa la voz (...). Si, vamos toditos, a todas nos convocan y todas vamos, somos como 50 vasos de leche en el distrito a todos nos mandan un oficio para ir a las charlas o las reuniones" (E7, L15-36).

Con respecto a la relación de lo asistencial con la participación comunitaria en salud, las entrevistadas primordialmente la focalizan con la labor asistencialista, de identificación y seguimiento de casos en comunidad que son diagnosticados en el establecimiento de salud, y es desde ahí que ellas agilizan los procesos de atención dentro del mismo establecimiento de salud y es por ello que sustentan su trabajo en la difusión de campañas, la socialización a los vecinos y que promuevan su asistencia a los eventos que se realicen donde se ofrecen servicios gratuitos y masivos además que los servicios de salud, como podología, entre otros:

"Lo que pasa es de que con el centro de salud en estas zonas no creo que sea mucho, que tengan que ir o acercarse al centro (...), pasa de que cuando hay campañas nos dicen: vayan, va a ver exámenes de laboratorio ¿no?, entonces todo tipo de atención para todas las especialidades, pero muchas veces se les da prioridad al que pertenece al centro" (E1, L106-121).

"Muchas veces cuando realizamos las campañas, cuando hay campañas se hace la convocatoria ¿no?, informarle a la gente, ya que el que no tiene seguro o no cuenta en sí que pueda llegar al centro, pueda ser atendido aquí ¿no? (...), lo que facilita en sí es la convocatoria masiva, es la información que se volantea, digamos te avisan de antes y nosotras que salimos a la calle estamos informando (...). En cuanto a la salud, pienso que las campañas, ¿por qué? Porque si no se enteran, si no sabe Ud. no va a salir de casa para ir a, que yo le diga vaya al centro, ¿para qué? Si no tienen una consulta, no se preocupan en sí por ahondar un poquito más en su salud (...)" (E1, L73-127).

En relación a la manera en que la comunidad debiera participar en salud, las entrevistadas perciben que debe fortalecerse el nivel de comunicación o difusión de los servicios asistenciales del establecimiento de salud, por lo que ellas centralizan las acciones en pro de la salud de la comunidad desde el direccionamiento del establecimiento de salud, constituyéndose en aliadas de fuerza en esta labor. Estos hallazgos concuerdan con lo propuesto por Valenzuela F et al (3) en el que los agentes comunitarios deben tener pleno conocimiento de su propia situación y deben contar con incentivos para resolver sus problemas comunes, permitiéndoles ser agentes de su propio desarrollo.

"Poniendo en comunicación o con afiches para que la gente tenga conocimiento de que hay un programa y que se pueden acercar" (E2, L37).

"Para elaborar el plan debe de llamar a las cabezas que somos las que estamos más enteradas que una socia, escucha lo que le decimos, pero una socia no camina como caminamos nosotras o sea nosotras tenemos otra clase de capacitación (...) entonces, yo les explico, pero les explico a como yo he entendido" (E8, L36).

Con respecto a la manera de identificar problemas de salud en la comunidad, las entrevistadas refieren que para su labor necesitan mayor apoyo en las capacitaciones, difusión, y en cada intervención que realizan, no lo hacen de manera individual, sino lo hacen de manera integral con toda la familia:

"Bueno, de repente un poquito más de apoyo en cuanto 
a visitas, visitas ya médicas, que haya por lo menos sobre la problemática, nosotros informar, informar, que nos ha pasado (...) preparamos un informe y comentar para que el médico vaya a visitarlo (...) que lo vayan a ver, vayan a realizar la visita y que se pueda ayudar con lo que se pueda ¿no?" (E1, L49).

"Poniendo en comunicación o con afiches para que la gente tenga conocimiento de que hay un programa y que se pueden acercar" (E2, L37).

\section{DISCUSIÓN}

De acuerdo a nuestros hallazgos, las expresiones evidenciadas concuerdan con lo descrito por Barrera $\mathrm{H}$. y Pacheco S. (17); Miranda V. y Mendoza M. ${ }^{(18)}$, quienes refuerzan el hecho de que la Planificación participativa de la comunidad permite organizar todos los esfuerzos de los agentes comunitarios y los orienta hacia el desarrollo comunal, basándose en una pertinente identificación de problemas comunitarios. Asimismo, los estudios de Anigstein C. (19), Cassetti V., López V., Paredes J. (6); Paño, Rébola, Suárez ${ }^{(7)}$, quienes definen la participación comunitaria como una interrelación entre los diferentes agentes comunitarios en una comunidad para lograr un objetivo común, mediante el uso de diversos métodos tanto de consulta, empoderamiento o colaboración. La participación que se evidencia es de tipo pasiva o pseudoparticipación, pues se da cuando las personas actúan a solicitud de otra persona, sin que pueda intervenir en la toma de decisiones ${ }^{(20)}$. Sin embargo, algunas participantes refirieron que su participación estaba más relacionada a la posibilidad de recibir capacitaciones, charlas sobre temas diversos de salud. Según Geilfus ${ }^{(21)}$, propuso la "Escalera de la Participación", en la cual lo descrito corresponde a la Participación por incentivos, es decir, cuando les brindan un incentivo no pecuniario a las personas que participan, pero sin tener opción para la toma de decisiones finales. Se evidencia nuevamente un fuerte componente de injerencia política, pues la vivencian en la intromisión en aspectos de salud de las autoridades locales a fin de conseguir adeptos a las autoridades locales y Regionales de turno, con un fin político-partidario, lo cual desvía el foco de la atención de la participación comunitaria ${ }^{(22)}$.

Sobre el compromiso por la participación comunitaria, en diversas experiencias nacionales e internacionales se muestra que los agentes comunitarios en el cumplimiento de sus funciones deben denotar compromiso individual y/o colectivo al asumir una responsabilidad por solucionar problemas comunes ${ }^{(23,24)}$, y que esas responsabilidades van a permitir que la participación comunitaria sea más evidente fomentando en las demás personas querer ser partícipes en ello ${ }^{(25,26)}$.

Las entrevistadas refirieron que conceptualizan el Empoderamiento como el fortalecimiento de su accionar mediante capacitaciones que les permitan ejercer una labor más sostenible en la comunidad, garantizando el seguimiento de casos de pacientes captados en el establecimiento de salud y/o en la comunidad, notándose que sigue habiendo la orientación predominantemente asistencialista y recuperativa de la atención, dándole en menor proporción a los aspectos preventivo-promocionales ${ }^{(27,28)}$. Esto concuerda con Sosa I. y colaboradores ${ }^{(29)}$; y Delgado M. y colaboradores ${ }^{(30)}$, sobre la Clasificación de la Participación de tipo Pasiva o Pseudo-participación. Esto como veremos, ha sido muy repetitivo a lo largo del desarrollo de las entrevistas realizadas en esta comunidad.

Si bien los autores coinciden en que la visita domiciliaria contribuye a fortalecer el abordaje de manera integral en el seno familiar y permite un mayor acercamiento de nuestro Sistema de Salud hacia la población que por algún motivo tiene algún tipo de problemas de accesibilidad, no necesariamente geográfica ni económica, sino de diversa índole, en esta comunidad de Bellavista se percibe que es una actividad más reactiva que proactiva. Sobre la ejecución de la participación, Gatti C. y colaboradores ${ }^{(31)}$; incide en que la participación puede ser visto como un medio, pues se usan recursos comunitarios existentes para lograr alcanzar las metas diseñadas, es decir priorizando los resultados, y como un fin pues resulta ser un crecimiento dinámico que responde a las necesidades identificas y las circunstancias del entorno cambiante.

Se llegó a la conclusión que el tipo de participación de la comunidad evidenciada es Pasiva o Pseudoparticipación. Se remarca la injerencia político partidaria en temas de salud. Se reconoce la intervención de los agentes comunitarios, por su labor con motivación, vocación de servicio y empatía, pero poseen un sesgo de su labor prioritariamente asistencialista en su relación con el establecimiento de salud. Se recomienda proponer el trabajo de fortalecimiento de capacidades para reorientar el Trabajo comunitario y todo el esfuerzo desplegado hacia la comunidad, cambiando la concepción asistencialista de los agentes comunitarios involucrados.

\section{REFERENCIAS BIBLIOGRÁFICAS}

1. Gómez E, Vásquez G, Lenti A, Franco L, Herrera G, Aguirre G, et al. Planeación Participativa: Realidades y Retos [Internet]. 2012. p. 1-300. Available from: http://www.ts.ucr.ac.cr/binarios/libros/ libros-000028.pdf

2. Ministerio de Salud. Planificación Local Concertada para el Desarrollo [Internet]. 2016. p. 1-41. Available from: http://bvs. minsa.gob.pe/local/MINSA/764_MINSA188.pdf

3. Salvatierra M. Percepciones sobre la participación comunitaria en un grupo de vecinos integrantes de un proyecto cultural ubicado 
en un barrio de Comas. [tesis maestría]. Lima-Perú: Universidad Antonio Ruiz de Montoya; 2019. [Internet]. Available from: http:// repositorio.uarm.edu.pe/bitstream/handle/20.500.12833/2076/ Salvatierra Fajardo\%2C María Pía_Tesis_Licenciatura_2019. pdf?sequence=1\&isAllowed=y

4. Valenzuela F, Salaverry O, Monteza B, Fuentes D. Percepción del Usuario sobre los servicios de salud de la comunidad de Chopcca, Huancavelica, desde una mirada Intercultural (Resultado Preliminar). Boletín INS [revista en Internet] 2015 [acceso 02 de noviembre de 2021]; 2021(1-2): 16-23. Available from: https:// repositorio.ins.gob.pe/bitstream/handle/INS/325/BOLETIN2015ene-feb-16-23.pdf?sequence=1\&isAllowed=y

5. Reiner L, Cruz B, Orozco C. Importancia de la participación ciudadana en la atención primaria. Edumecentro [revista en Internet] 2019 [acceso 02 de noviembre de 2021]; 11(1): 218-233. Available from: http://scielo.sld.cu/pdf/edu/v11n1/2077-2874edu-11-01-218.pdf

6. Cassetti V, López V, Paredes J. Participación comunitaria: Mejorando la salud y el bienestar y reduciendo desigualdades en salud. [Internet]. 2018. p. 1-66. Available from: https://portal. guiasalud.es/wp-content/uploads/2019/01/GPC_579_Guia_ Adapta_Participacion_-Comunitaria.pdf

7. Paño P, Rébola R, Suarez M. Procesos y Metodologías Participativas: Reflexiones y experiencias para la transformación social [Internet]. 2019. p. 1-434. Available from: http://biblioteca.clacso.edu.ar/ clacso/gt/20190318060039/Procesos_y_metodologias.pdf

8. Sefrin K, Alberto I, de Lima G, Soares S. Percepções dos agentes comunitários de saúde: contribuições para a gestão em saúde. Revista CUIDARTE [revista en Internet] 2016 [acceso 01 de noviembre de 2021]; 7(2): 1325-1337. Available from: https:// revistas.udes.edu.co/cuidarte/article/view/338/731

9. Ministerio de Salud del Perú. Orientaciones para el fortalecimiento de la labor del agente comunitario de salud: Documento Técnico [Internet]. 2014. p. 1-37. Available from: http://bvs.minsa.gob.pe/ local/MINSA/3154.pdf

10. Borda A, Palma M, do Amaral J. Percepciones de gestores de salud y facilitadores de la estrategia Atención Integrada a las Enfermedades Prevalentes (AIEPI) en una zona del Noreste de Brasil y Perú. Interface [revista en Internet] 2017 [acceso 02 de diciembre de 2021]; 21(63): 933-943. Available from: https://www.scielo.br/j/ icse/a/TWYyWZZthYYcnJKv7sNzndc/?format=pdf\&lang=es

11. Montalvo J, Villanueva Y, Gómez E, Cervantes Y. Participación comunitaria y responsabilidad social universitaria: estudio de caso. Revista Global de Negocios [revista en Internet] 2017 [acceso 02 de noviembre de 2021]; 5(5): 99-111. Available from: https:// papers.ssrn.com/sol3/papers.cfm?abstract_id=2916377

12. Sánchez J, Arredondo M, Leyva C, Ávila G, Figueroa C, Mata J. Participación comunitaria y percepción social en Latinoamérica: un futuro para las áreas protegidas y proyectos de geoparques. Ambiente y Desarrollo [revista en Internet] 2018 [acceso 02 de noviembre de 2021]; 21(41): 61-77. Available from: https:// dialnet.unirioja.es/servlet/articulo?codigo $=6563371$

13. Hernández-Sampieri $R$, Mendoza C. Metodología de la investigación. Las rutas cuantitativa, cualitativa y mixta. México: Mc Graw-Hill; 2018. 753 p.

14. Grimaldo M. Manual de Investigación en Psicología. Capítulo: Investigación Cualitativa [Internet]. 2009. Available from: https:// www.academia.edu/14147106/Manual_de_Investigación_en_ Psicología_Capítulo_Investigación_Cualitativa

15. Gómez P. Principios básicos de bioética. Revista Peruana de Ginecología y Obstetricia [revista en Internet] 2009 [acceso 25 de abril de 2021]; 55(4): 230-233. Available from: http://www.spog. org.pe/web/revista/index.php/RPGO/article/view/297/268

16. Carreño J. Consentimiento informado en investigación clínica: un proceso dinámico. Persona y bioética [revista en Internet] 2009 [acceso 01 de diciembre de 2021]; 20(2): 233-243. Available from: http://www.scielo.org.co/pdf/pebi/v20n2/0123-3122pebi-20-02-00232.pdf

17. Barrera $\mathrm{H}$, Pacheco S. Planeación participativa como una alternativa de desarrollo. Ciencia Y Agricultura [revista en Internet] 2013 [acceso 01 de noviembre de 2021]; 10(2): 29. Available from: https://revistas.uptc.edu.co/index.php/ciencia_agricultura/ article/view/2838/2606

18. Miranda V, Mendoza M. Programa de formación de líderes comunitarios(as): Diagnóstico y Planificación Comunitaria [Internet]. 2006. p. 1-78. Available from: http://www. asocam.org/sites/default/files/publicaciones/files/ ff203078ec818bb075fc4869c7638334.pdf

19. Anigstein C. Gestión Participativa y Salud: evaluación de Municipio de General Rodríguez [tesis maestría]. Buenos Aires-Argentina: Instituto Universitario ISALUD; 2004. [Internet]. 2004. Available from: http://repositorio.isalud.edu.ar/xmlui/handle/1/224

20. Delgado M. Evolución del conocimiento del sistema de salud, derechos en salud, de la política de participación y de las experiencias de participación de los usuarios del Sistema General de Seguridad Social [tesis doctoral]. Bellaterra-España: Universitat Autònoma de Barcelona [Internet]. Available from: https:// www.tdx.cat/bitstream/handle/10803/125907/medg1de1. pdf?sequence=1\&isAllowed $=y$

21. Geilfus F. 80 Herramientas Para el Desarrollo Participativo [Internet]. 2002. p. 1-218. Available from: http://tie.inspvirtual. $\mathrm{mx} /$ recursos/temas/etv/OAParticipacionSocialWeb/material/80 herramientas para el desarrollo participativo.pdf

22. Amar J, Echeverría J. Participación comunitaria para el control ciudadano de los servicios públicos domiciliarios. Revista de Derecho [revista en Internet] 2008 [acceso 02 de noviembre de 2021]; 29(1):129-152. Available from: http://www.scielo.org.co/ pdf/dere/n29/n29a06.pdf

23. Montero M. El fortalecimiento en la comunidad, sus dificultades y alcances. Intervención Psicosocial [revista en Internet] 2004 [acceso 02 de noviembre de 2021]; 13(1):4-19. Available from: https://www.redalyc.org/pdf/1798/179817825001.pdf

24. Sossa A, Olivos F, Brange A. Participación comunitaria, confianza y percepción de derechos en Latinoamérica y el Caribe: evidencia de 18 países. Revista nuestrAmérica [revista en Internet] 2019 [acceso 02 de noviembre de 2021]; 7(14): 228-257. Available from: https://dialnet.unirioja.es/servlet/articulo?codigo=7061492

25. Cueto R, Seminario E, Balbuena A. Significados de la organización y participación comunitaria en comunidades vulnerables de Lima Metropolitana. Revista de Psicología [revista en Internet] 2015 [acceso 02 de noviembre de 2021]; 33(1): 57-86. Available from: http://www.scielo.org.pe/pdf/psico/v33n1/a03v33n1.pdf

26. Delgado $M$, Vázquez $M$. Percepciones de usuarios y líderes comunitarios sobre su capacidad para influenciar en la calidad de los servicios de salud: un estudio de casos de Colombia y Brasil. Cad. Saúde Pública [revista en Internet] 2009 [acceso 02 de noviembre de 2021]; 25(1): 169-178. Available from: https://www.scielo.br/j/ csp/a/tYQX3mGRDWwXpSSpvvRjFdM/?format=pdf\&lang=es

27. Rojas R. El liderazgo comunitario y su importancia en la intervención comunitaria. Psicología para América Latina [revista en Internet] 2013 [acceso 02 de noviembre de 2021]; 25(1): 57-76. Available from: http://pepsic.bvsalud.org/pdf/psilat/n25/a05.pdf

28. Delgado $M$, Vázquez L. Barreras y Oportunidades para la Participación Social en Salud en Colombia: Percepciones de los Actores Principales. Revista de salud pública (Bogotá, Colombia) [revista en Internet] 2006 [acceso 02 de noviembre de 2021]; 8(2): 128-140. Available from: https://www.scielosp.org/pdf/ rsap/2006.v8n2/128-140/es

29. Sosa I, Lefévre P, Guerra M, Ferrer L, Rodríguez A, Bonet $M$, et al. Propuesta metodológica para la planificación, implementación y 
evaluación participativas en Áreas de Salud - Consejos Populares. Revista Cubana de Medicina General Integral [revista en Internet] 2013 [acceso 01 de noviembre de 2021]; 29(2): 173-183. Available from: https://www.medigraphic.com/pdfs/revcubmedgenint/cmi2013/cmi132h.pdf

30. Delgado $M$, Vázquez $M$, Zapata $Y$, Hernán M. Participación social en salud: Conceptos de usuarios, líderes comunitarios, gestores y formuladores de políticas en Colombia. Una mirada cualitativa. Revista Española de Salud Pública [revista en Internet] 2005 [acceso 01 de noviembre de 2021]; 79(6): 697-707. Available from: https://scielo.isciii.es/pdf/resp/v79n6/original6.pdf

31. Gatti C, Villalba R, Molina G, Alegría H. Salud y Participación Comunitaria [Internet]. 2017. p. 1-184. Available from: http://iah. salud.gob.ar/doc/Documento175.pdf 